\title{
Mitigating safety risk through confidential reporting
}

\author{
C. Langer \\ CIRAS (Confidential Incident Reporting and Analysis System), UK
}

\begin{abstract}
CIRAS has been in business for 18 years. This paper demonstrates how unique insights from confidential reporting can prevent accidents, helping to improve organisational resilience - and, ultimately, business performance. CIRAS is able to 'trap' information from frontline staff who choose for a variety of reasons to use an independent service. This information might otherwise be lost by conventional, internal reporting systems because in certain circumstances, they may not be fully utilised. CIRAS is therefore in a position to share why some staff feel that internal reporting channels have not been successful, or in a minority of cases have come directly to CIRAS. The barriers to safety reporting, why staff choose to report to CIRAS, and the positive changes facilitated by CIRAS reports are all discussed here. In addition, the safety lessons and insights gained predominantly from the rail industry are directly applicable in many other industries too. Confidential reporting can, in fact, work very effectively in any industry where there are safety risks, as the core principles are the same, regardless of the specific industry. A confidential reporting system such as CIRAS can also help demonstrate how an organisation can enhance an already strong safety culture by promoting and exploiting the opportunities for learning. Confidential reports can help an organisation focus on what it does well - and not so well - and make the best use of its existing resources. Where weaknesses in safety defences are identified, such confidential reports offer an opportunity for the organisation to act maturely enough without feeling that such reports challenge its own authority. Moreover, they also present an organisational learning opportunity, not only to facilitate the resolution of such reports, but also to learn and understand what else went wrong within its own internal reporting processes. Over its lifetime, CIRAS has in fact facilitated the resolution of many thousands of safety issues. By taking the approach that a willingness to report apparently minor safety issues, as well as higher risk ones, CIRAS believes that
\end{abstract}


the organisational willingness to embrace confidential reporting serves as another line of defence in safety strategy and risk control. Providing appropriate, timely feedback to every reported safety concern is the cornerstone of an effective safety culture.

Keywords: confidential reporting, safety culture, accident prevention, risk management, internal reporting, organisational resilience, feedback, learning.

\section{Introduction}

This paper highlights the benefits of confidential reporting to industries where safety is of paramount importance, but also to other industries where the management of risk is a key driver. It details how the implementation of confidential reporting can mitigate safety risks through the resolution of long-standing issues which have not been fully addressed through internal reporting channels.

The Confidential Incident Reporting and Analysis System (CIRAS) began life in the UK as a pilot scheme at ScotRail in 1996. The experience of CIRAS within the rail industry in the last 18 years demonstrates how mature organisations across the whole industry continue to benefit, not just in safety terms, but in terms of organisational learning and resilience, and business performance too.

In 2014, CIRAS will launch its strategy for the next five years and this recognises the many learning opportunities that are available to CIRAS. Many of the owning groups that operate within the UK rail industry have other transport modes as part of their transport portfolios. As a result, CIRAS will provide its service to other transport interests such as marine, highways, aviation, coach and bus and other rail systems - for example, tram and light railway. Reporting behaviours and how a company responds are clearly indicative of its culture.

The more organisations that embrace CIRAS, the more we can learn about the safety behaviours and culture that cross multiple transport modes, even within the same corporate organisations. Confidential reporting is not new. It is already utilised in many industries beyond rail. Serving as a strong model of good practice, CIRAS continues to evolve by offering companies a corporate safety net and last line of defence and is indicative of a strong safety culture. It is argued here that confidential reporting is a key determinant of a strong safety culture. Its universal adoption across the rail industry shows how an independently operated scheme can contribute to improved lines of safety defence within an organisation's safety management system, as well as providing a proactive way to learn from potential weaknesses, in cultural and safety and terms. For some organisations, confidential reporting is simply seen as an integral component of their safety management systems - this is actively encouraged by CIRAS.

It is extremely difficult to say with any degree of certainty what CIRAS reports have actually prevented an accident from occurring. However, we can say unreservedly that confidential reporting through CIRAS has made the rail 
industry an even safer place. The actions taken by member organisations over the years clearly demonstrate that reporting employees have contributed to a safer working environment. Those organisations responding positively are well on their way to achieving a strong, open safety culture.

\section{Brief history of CIRAS}

CIRAS began in 1996 when a team from Strathclyde University was asked to introduce a confidential reporting system for ScotRail. Soon participation in the system had spread to a number of railway organisations in Scotland. Other railway companies started to show interest in an industry wide confidential reporting system for health and safety matters. Following the Ladbroke Grove incident in 1999, CIRAS was extended to include the whole of the mainline rail system in Great Britain. Today, CIRAS is now open to other forms of transport including coach and bus, marine, light railway including trams and aviation.

\subsection{From pilot scheme to national system}

CIRAS existed first as a pilot scheme, operated by ScotRail. The University of Strathclyde ran the scheme to help preserve its independence. Confidential reporting was recognised as an important way of surfacing human factors data that might otherwise be missed by conventional reporting channels. As Davies et al. [1] point out:

'...existing reporting channels are often associated with disciplinary action, and this distorts both the nature and number of reports received. This is particularly true in the railway industry where, historically, relationships between workforce and management have sometimes been characterised by mutual mistrust and animosity, rather than co-operation.'

At the time, the purported aims of the system were as follows: firstly, to collect reports from safety critical staff such as drivers, signallers and track workers which might not normally be reported through conventional channels, and secondly, to enhance existing safety management systems. The intention has always been to complement existing reporting channels, not replace them in any way.

Before CIRAS began as a pilot scheme, CHIRP (Confidential Human Factors Reporting Programme) had already been operating in the aviation industry for over a decade. Their mode of operation provided the model for CIRAS. There were many similarities between the two systems which still exist today. Both systems continue to take reports confidentially, but not anonymously (meaning that the individuals reporting are known to CIRAS), and both publish their own newsletter.

In June 2000, CIRAS obtained a national mandate for its operation as a charitable trust, extending its coverage to all UK Railway Group members. This 
followed the Ladbroke Grove train accident, where 31 people lost their lives, and the subsequent Cullen Inquiry, which encouraged the scheme's extension [2]:

'A confidential reporting system, CIRAS, is now in place across the industry. It is to be hoped that in the longer term the culture of the industry would be such as to make confidential reporting unnecessary. I accept that this situation may be a long time in coming to pass in the industry. In the meantime I fully support and encourage the further use of the CIRAS system.'

At this point in time, CIRAS began to cover every train and freight operating in the UK, the Infrastructure Manager (Network Rail), the vast majority of contracting companies and London Underground. It became possible for anyone working in the railway industry to report a safety concern, in recognition of the fact that reports from non-safety critical staff could yield important safety information too.

\subsection{Recent developments}

CIRAS was formally incorporated into Rail Safety Standards Board (RSSB) in 2008, giving up its status as a charitable trust. It remains operationally independent with its governance overseen by a committee comprising member organisations and various independent persons, including its own chairman. Despite this operational independence, there are now greater in-house opportunities for the sharing of railway intelligence between CIRAS and RSSB.

In September 2010, the new 'Rail Industry Standard for a Confidential Reporting System for Rail Staff' [3] came into effect. Though there was no longer any mandatory requirement for Railway Group members to subscribe to CIRAS, despite the fears in some quarters, no existing members withdrew their support. In fact, CIRAS continues to grow, taking on new members from the various sectors of the transport community.

CIRAS extended its reach into other transport areas in January 2014. This allows us to engage other sectors such as marine, light railway, trams and bus, aviation and other transport related modes. The purpose of this is to create a learning hub for cultural and safety reporting issues.

\section{Who uses CIRAS?}

Originally, CIRAS only took reports from safety critical staff. These days, CIRAS will take reports from any rail staff or other transport members with a genuine safety concern. However, it is clear from the chart below (Chart 1) that safety critical staff - such as drivers (29 per cent), track workers (13 per cent), station staff ( 10 per cent), conductors ( 6 per cent) and signallers ( 6 per cent $)-$ still tend to report more than other categories. 


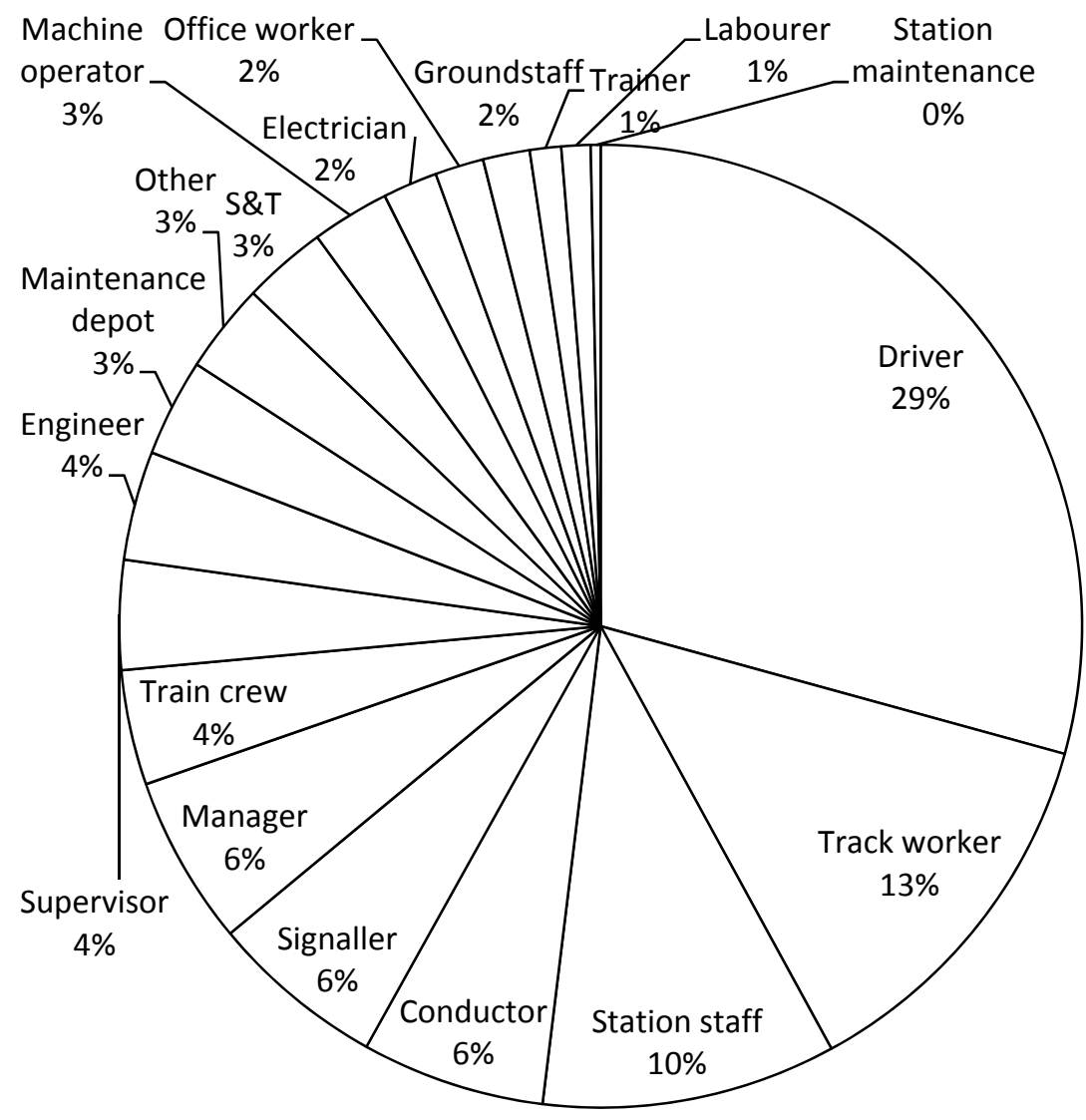

Figure 1: Chart $1-$ who uses CIRAS?

\section{Why do staff use CIRAS?}

The most common reason for staff coming to CIRAS with a safety concern is because they feel an issue at work has been left unresolved. In the vast majority of cases ( 81 per cent), their safety concern has already been reported internally but the response has been perceived as unsatisfactory in some way.

The pie chart (Chart 2) below shows the reporters' perceptions of the response they have received through their internal reporting channels. However, we are looking to expand the categories for why staff use a confidential reporting 
system - for example, interpersonal conflict, culture, and poor industrial relations at work may all feature in the decision to approach CIRAS.

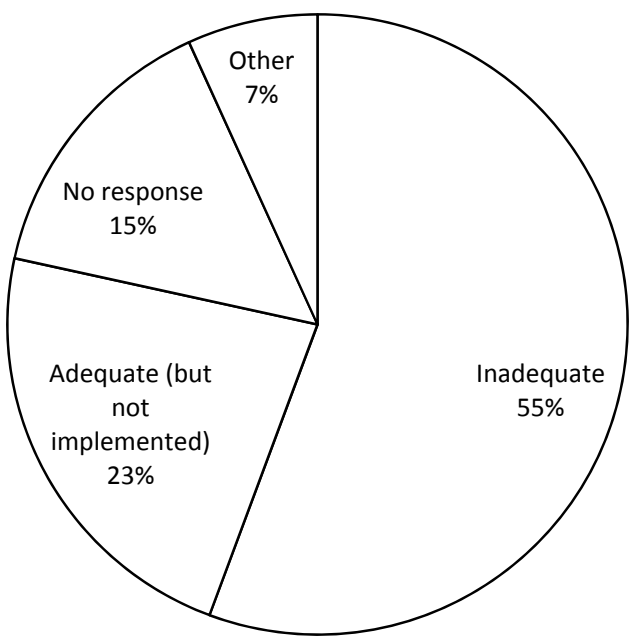

Figure 2: Chart 2 - perceptions of internal response to safety concern.

\section{Positive outcomes}

Positive outcomes from CIRAS reports can be analysed in a number of different ways. This section considers the data both quantitatively and qualitatively.

\subsection{Quantitative outcomes}

Over a 30 month period between May 2011 and November 2013, all our returned evaluation forms from companies receiving reports were analysed. The return rate for these forms is particularly high, standing at 74 per cent for 2013.

Each responding company is asked what actions they have taken in relation to the CIRAS report they received. Companies are advised to tick as many actions as apply in their particular case. In total over this period, 911 actions were taken by companies in relation to the CIRAS reports they received. The chart below shows how these actions have been categorised.

'Monitoring', which may be thought of as a positive form of attention on a safety issue, accounted for 24 per cent of cases, followed by the category 'Planned action' (18 per cent) which denotes the intention to carry out a safety-related action in the future.

Though it could be argued these categories are a little vague and difficult to measure in reality, many of the other categories represent more substantial actions. For example, the next two categories, 'Investigation' (15 per cent) and 'Briefing' (12 per cent) may have a considerable impact on safety related behaviour. Though relatively low in percentage terms, some CIRAS reports have 
led to actions which are likely to have had a considerable impact on safety. For example, in 4 per cent of cases an audit was undertaken; in 3 per cent of cases, safety equipment was provided; in 2 per cent of cases, infrastructure faults were attended to; in 2 per cent of cases, training was provided. The full breakdown of these results is shown in Chart 3 below.

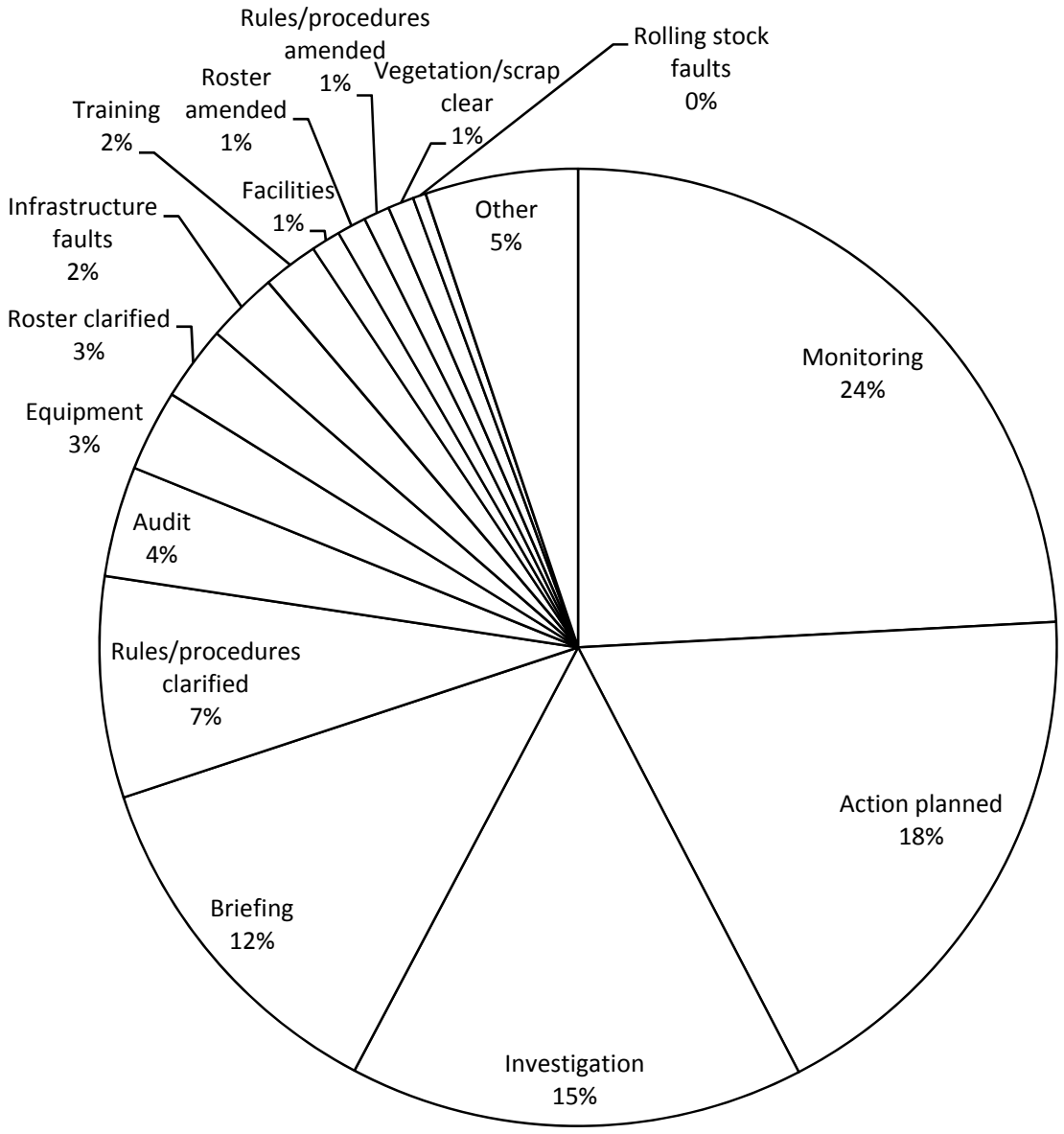

Figure 3: Chart 3 - breakdown of outcomes from CIRAS reports.

\subsection{Some qualitative data: examples of action taken}

CIRAS actively tracks what the outcomes of its reports are by directly asking the respondents of reports what has changed. These outcomes have been discussed already in quantitative terms above. However, in qualitative terms, it is helpful to point to some specific subject areas of reporter concern and how these have been 
addressed. The following list of outcomes from the last 12 months is by no means exhaustive, but is a fairly representative selection of the issues handled by CIRAS. All the reports listed here were highly rated by the respondents.

- A full audit and monitoring of several practices perceived as unsafe at a major contractor were highlighted. Communication was improved and further training and briefing were provided.

- Contradictory signage on a part of the West Coast mainline was rectified following a full investigation. Other geographically adjacent areas were also checked for similar issues.

- A non-compliant coach lifting procedure at a train operating company's depot was addressed. The practice immediately ceased and correct process implemented. Improved briefing was also introduced.

- Shunting procedures in a large depot were reviewed by a train operating company and improved briefing provided.

- Station dispatch arrangements were reviewed comprehensively by a train operating company with a specially arranged site test. Improvements have been identified.

- A lack of training and protective clothing for staff were highlighted, leading to an investigation and the subsequent training, briefing and provision of protective clothing by a major contractor.

- The highlighting of scrap rail left on the infrastructure and causing a safety hazard led to an investigation and its subsequent removal.

- The highlighting of high dust levels coming from a ballast cleaner led to a range of control measures being implementing by a major contractor, including better equipment and enhanced training and briefing.

- The reporting of long travel times for subcontracting staff led to increased monitoring, briefing and the provision of facilities.

- Limited feedback to safety reports made internally was being provided by a train operating company. Following the issue being raised by CIRAS, the feedback process was reinstated.

\section{Organisational benefits}

There are considerable benefits for an organisation subscribing to a confidential reporting system such as CIRAS. Some of the main ones are described here.

\subsection{Jogging organisational memory}

Confidential reporting provides an opportunity for organisations to explore the link between past safety incidents and the potential for something similar to happen in the future. It helps safety organisations tap into their memory banks, and review their rules and operating procedures.

For an organisation to make a change, it has to first review what isn't working and what it needs to do to improve and therefore change. Good organisations will review past incidents to reflect, identify, learn and implement corrective 
action and improvement. A confidential report often serves to kick-start this process of reviewing safety management systems. In short, it often becomes an important catalyst in raising concerns that the organisation might otherwise ignore.

Confidential reporting encourages organisations to retrieve safety critical experience from their memory banks - and then reflect on it. Safety lessons from an organisation's past can be brought to bear on safety decisions in the present. It is an effective management safety tool with the power to inform decision-making

\subsection{Nudging management behaviour}

The 'nudge' approach has been popularised by Thaler and Sunstein's [4] book of the same name. CIRAS can often nudge a company into re-thinking its approach to a long-standing issue that hasn't yet found resolution. Confidential reporting often proves itself able to nudge a company into responding differently using a purely facilitative approach.

The overwhelming majority of reports to CIRAS have been reported through company reporting channels before reaching CIRAS. Yet over 70 per cent of these reports will still prompt, or nudge, a form of positive action from the company concerned. So in most cases, the company has already been made aware internally of the particular issue. However, for whatever reason - lack of appreciation of the risks involved, local politics, or general inertia - the issue has remained unresolved. There is something about receiving a confidential report which frequently makes the difference. As stated earlier, good organisations see a confidential report being an opportunity to address an issue rather than a threat to its own authority.

As a facilitator, CIRAS can never act coercively or enforce actions like a regulator can. Nevertheless, a lack of regulatory power can prove to be an advantage. Nudging managers into re-thinking their standard responses is likely to be more effective without regulatory input which may encourage merely reactionary responses. For managers keen on learning from reporting, it is more about proactively reviewing the current safety management system and spotting potential weaknesses before a safety incident takes place.

There can be little doubt that CIRAS helps different companies within a sector learn from each other. Within a particular sector, companies often face very similar safety issues. By publishing a newsletter with reports and their responses, CIRAS aims to promote learning and exploit the opportunity for improved understanding of the cultural issues that often influence internal reporting and its success rates.

\subsection{Highlighting unacknowledged safety risks}

Over the 30 month period under analysis (May 2011-November 2013), there are several indications that CIRAS manages to 'trap' information that otherwise would be lost. Companies responding to CIRAS reports are asked whether any information if the report contains new information. 
The consistent, long-term trend is for over 70 per cent of reports to be channelled internally before ending up at CIRAS. Yet 35 per cent of CIRAS reports still contain new information (as confirmed by the companies themselves). It is therefore fair to argue that a large chunk of credible safety information is being lost internally, but is then later captured by CIRAS.

\subsection{Preventing accidents: being alert to 'black swans'}

The main driver for providing CIRAS with a national mandate for operation in 2000 was the Ladbroke Grove accident where many people lost their lives. The consensus was that confidential reporting could play an important role in preventing a similar accident in the future.

Essentially, what CIRAS is doing in presenting new information and in doing so is providing the opportunity to address an unacknowledged safety risk. It is clear that this new information is frequently viewed as very valuable - it can help mitigate these unseen risks.

What an organisation doesn't know can be a significant contributor to safety risk. Until a safety incident actually happens on a scale worthy of note, it may not appear prominently at all on the 'safety radar'.

Safety risk models invariably use data from known safety incidents, but cannot adequately account for unpredictable safety events which can have a far greater impact. Confidential reporting helps highlight potential events and patterns which have not yet manifested themselves as fully-blown safety events. The information from confidential reports can be a critical tool in determining where safety managers focus their attention. It is just as important to be strategically alert to new safety risks, as it is to focus on data from old incidents.

This approach is broadly in line with Taleb's [5] approach in 'The Black Swan' about the impact of highly improbable events where the unknown is considered to be even more relevant than the known. Hence, a low probability, but high impact event can have a major impact on the shape of an industry for years to come. Ladbroke Grove was one such example, leading to the widespread adoption of the Train Protection and Warning System designed to prevent that very accident from ever happening again.

\section{Conclusion}

The data presented here shows clearly how information provided in confidential reports is used to good effect in mitigating safety risks. Though all the data pertains to the railway industry, the same principles can be applied in other industries too. As CIRAS expands into other transport industries, it will be increasingly possible to extrapolate the lessons from one industry and apply them to another.

Confidential reporting has the clear potential to complement existing safety management systems and, when used effectively by mature safety organisations, can facilitate the healthy resolution of long-standing safety issues. 
In a significant proportion of cases, it can also highlight emergent safety risks. Such safety risks, which largely remain hidden and unacknowledged, will not feature in standard risk models.

Confidential reporting has an obvious role in surfacing such emergent safety risks in the pursuit of even fewer safety incidents and improved industry safety culture.

\section{References}

[1] Davis, J., Ross, A., Wallace, B. and Wright, L., Safety Management: A Qualitative Systems Approach. London: Taylor and Francis, 2003.

[2] Cullen, W.D. The Ladbroke Grove Railway Inquiry: Part 1 Report, 2001. http://www.railwayarchive.co.uk/documents/HSE_Lad_Cullen001.pdf

[3] Rail Industry Standard for a Confidential Reporting System for Rail Staff. RSSB, 2010. http://www.rgsonline.co.uk/Railway_Group_Standards/Traffic $\% 20$ Operation $\% 20$ and $\% 20$ Management/Rail\%20Industry\%20Standards/RI S-3701-TOM\%20Iss\%201.pdf

[4] Thaler, R.H. and Sunstein, C.R., Nudge: Improving Decisions about Health, Wealth and Happiness. Cornwall: Yale Book, 2008.

[5] Taleb, N.N., The Black Swan: The Impact of the Highly Improbable. London: Penguin, 2007. 